\title{
Large crane real-time safety assessment method based on Mamdani fuzzy inference system
}

\author{
Jian Chen $^{\mathrm{a}}$ \\ School of Electro-MechanicalEngineering, Guangdong University of Technology, Guangzhou510006, Guangdong, \\ China
}

\begin{abstract}
To build a state space evaluation system based on online sensor measuring information, combined with expert experience, a real-time safety assessment method based on Mamdani fuzzy inference system is developed, aiming to monitor safety of large crane in operation and accordingly provide decision support for crane operators. The assessment index system consists of crane's horizontal tilt angle, hook deflection angle and sidewise bending of boom, so the method can assess safety state of the crane in operation from view of the crane's space position. Making a 400ton crawler crane as an experiment object, real-time safety state of crane is evaluated. The result shows that the method described above is of availability, real-time capability and flexibility.
\end{abstract}

Keywords: assessment index; safety assessment method; fuzzy inference; real-time state; large crane in operation.

\section{Introduction}

With more and more cranes used in large-scale engineering construction, more related safety accidents also happened. The major safety accidents in these construction projects, on the one hand it's due to the user's inappropriate operation and management irregularities and other reasons, on the other hand, it is mainly due to lack of monitoring of safety state of the crane hoisting process. At present, on domestic cranes generally only crane torque limiter and lifting weight limiter are installed, and it is far from enough to prevent crane from accidents. Factors that affect safety also comprise hook deflection, crane's horizontal tilt, the sidewise bending of boom and wind speed etc..If these parameters can be monitored in real time, and safety evaluation results based on language description can feedback to the driver, it will certainly reduce the rate of accidents.

This paper selects three dynamic indexes of crane in lifting process for monitoring objects, namely the hook deflection angle, crane's horizontal tiltangle and the sidewise bending of boom. With the help of fieldbus technology, modern sensor technology and wireless network transmission technology, it is easy to implement real-time monitoring of the three parameters, but the operator is often unable to make decision quickly according to monitoring data, especially the crane is in dangerous working condition. Therefore, how can intuitively reflect the real-time safety state of the crane in lifting process is particularly important, and it is also the focus and innovation point of our research. Because of the strong nonlinearity, time variation and randomness of working condition of the crane during

${ }^{\mathrm{a} C}$ Corresponding author : chenjian7681@163.com

(C) 2016. The authors - Published by Atlantis Press 
hoisting process, traditional evaluation method based on precise mathematical model is difficult to describe safety status of the crane in real time. Fuzzy logic depends on exact mathematical description of the controlled object or process, showing a good application prospect in the applications of some highly nonlinear, uncertainty and time varying characteristics. In view of successful application of fuzzy logic in many security fields, this paper proposes a real-time safety evaluation method of crane based on fuzzy inference system of Mamdani type, trying to provide a solution for solving crane system safety evaluation out of structure[1][2][3].

\section{Principle of Mamdani type fuzzy inference system}

Mamdani type fuzzy inference system is a set of inference rules prior to the calculation to realize the inference from input to output[4][5].A single piece of generalized hypothetical reasoning can be expressed as:

Premise 1 (fact): $X$ is $A^{\prime}$.

Premise 2 (rule):if $x$ is $A$,then $y$ is $B$.

Succedent(conclusion): $\mathrm{y}$ is $B^{\prime}$.

\section{Real time safety evaluation model of crane based on Mamdani fuzzy inference system}

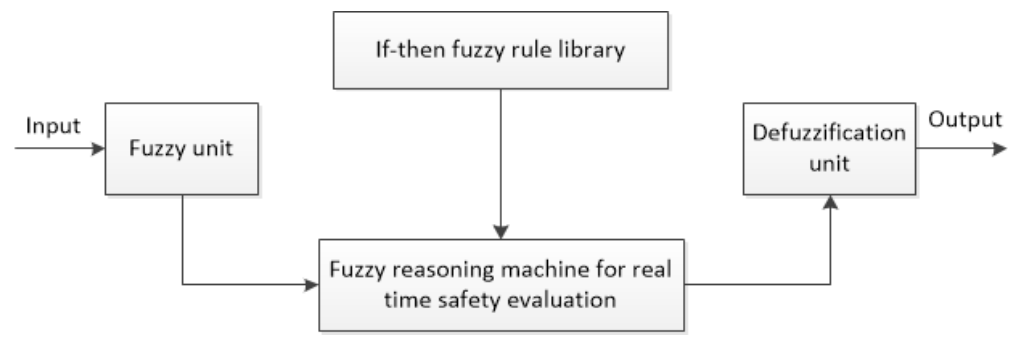

Figure1. General structure of crane real-time safety assessment model

\subsection{General structure of fuzzy inference system and choice of the input and output variables}

General structure of fuzzy inference system is shown in Fig.1. Through theoretical analysis and expert experience, this paper selects three parameters, which are the hook deflection angle, crane's horizontal tilt angle and the sidewise bending of boom, as monitoring objects which will be used by safety evaluation model with three evaluation indexes[1][2][3].Because of close relationship between the indexes and crane safe state, any one of the indexes exceeding given limit value may lead to major accidents (crane overturning or boombreaking). Therefore, the evaluation index system can objectively and comprehensively reflects real-time safety state of the crane. Because they can be easily obtained, data processing workload is small.

(1)Hook deflection angle $\alpha$. This variable reflects the real time position of the hook and affects safety of the crane.

(2)Horizontal tilt angle of the crane $\beta$. This variable reflects the overall level of the crane in the lifting process. If $\beta$ is beyond safe range it may cause the crane overturning, so it is necessary to monitor it to grasp overall horizontal level of the crane. Smaller $\beta$ is, Securer the crane is.

(3)Sidewise bending of the boom $\gamma$. This variable reflects deviation of the top of the crane during hoisting process in real time. If $\gamma$ is beyond safe range, it may cause the boom break. So, it is necessary to monitor sidewise bending of the boom in real time. Smaller $\gamma$ is, Securer the crane is.

(4)Crane's real-time safety level $Q$. Using Mamdani fuzzy inference system to deal with the above 
three input variables in real time, finally the output fuzzy quantity is transformed into corresponding real-time security level of the crane. Q is divided into three levels: security, warning, and danger.

\subsection{Determine the membership function of the input and output variables}

Technical parameters in Table 1 are provided by a mechanical lifting engineering company. All three zinput variables are divided into three fuzzy levels, namely, \{low, medium, high\}, and the output variable is divided into fuzzy level \{security, warning, danger\}.

Table1. Monitoring parameters and their values

\begin{tabular}{llll}
\hline Parameter & Safety value & Warning value & Danger value \\
\hline hook deflection angle $\alpha$ (degree) & $0 \sim 0.6$ & $0.6 \sim 1.2$ & $>1.2$ \\
horizontal tilt angle of the crane $\beta$ (degree) & $0 \sim 0.3$ & $0.3 \sim 0.57(1 \%$ & $>0.57$ \\
& & inclination $)$ & $>1.0$ \\
sidewise bending of the boom $\gamma($ degree $)$ & $0 \sim 0.5$ & $0.5 \sim 1.0$ & $>8 \sim 12$ \\
Crane real-time safety grade Q & $0 \sim 4$ & $4 \sim 8$ & \\
\hline
\end{tabular}

The selected input variable membership function of $\alpha, \beta$ and $\gamma$ is trapezoidal distribution function, the trapezoidal distribution curve represents a vector $x$ 's function, which depends on four scalar parameters, set to a,b,c,d. The function expression is as follow:

$$
f(x ; a, b, c, d)= \begin{cases}0, & x \leq a \\ \frac{x-a}{b-a}, & a \leq x \leq b \\ 1, & b \leq x \leq c \\ \frac{d-x}{d-c}, & c \leq x \leq d \\ 0, & d \leq x\end{cases}
$$

The membership function of output variable Q uses triangular distribution function. The triangle distribution function curve represents a function of the vector $\mathrm{x}$, which depends on three scalar parameters, set to a,b,c. The function expression is as follow:

$$
f(x ; a, b, c)= \begin{cases}0, & x \leq a \\ \frac{x-a}{b-a}, & a \leq x \leq b \\ \frac{c-x}{c-b}, & b \leq x \leq c \\ 0, & c \leq x\end{cases}
$$

Based on analysis of status of the hoisting process of large cranes and experience of engineers, the membership functions of the input and output variables are determined by Mamdani type fuzzy inference system in MATLAB, which are shown in Fig 2 to Fig5.

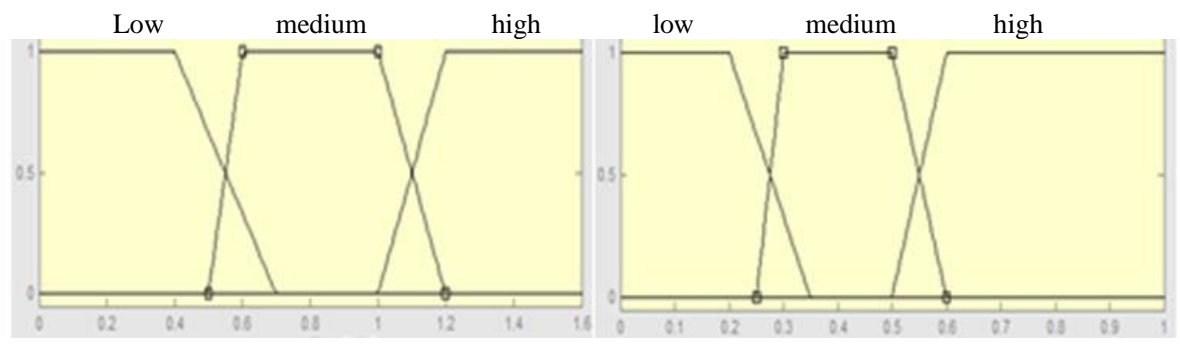

Figure2. Membership function of input variable $\alpha$ Figure3. Membership function of input variable $\beta$ 


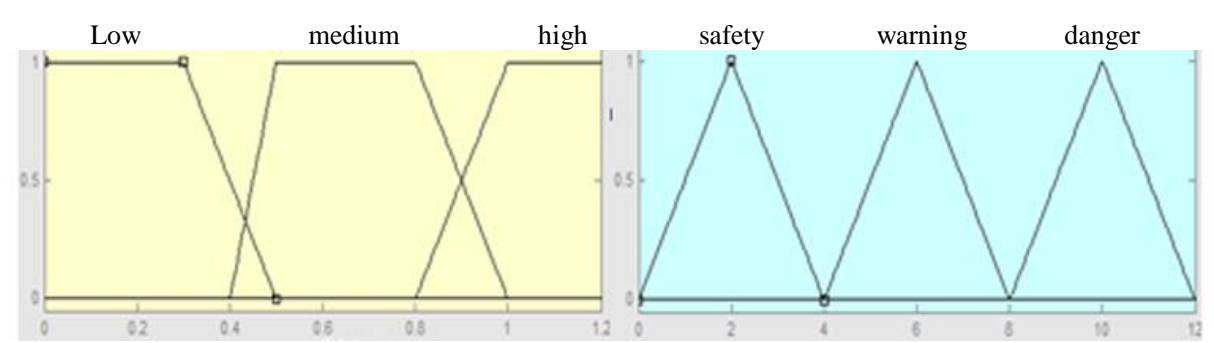

Figure4. Membership function of input variable $\gamma$ Figure5. Membership function of output variable Q

\section{Fuzzy reasoning method for real time safety evaluation model of crane}

\subsection{Determination of Mamdani type fuzzy inference if-then rules}

If-then rules are determined as following:

Rule 1: if ( $\alpha$ is high) or ( $\beta$ is high) or ( $\gamma$ is high) then ( $Q$ is danger).

Rule 2: if ( $\alpha$ is low) or ( $\beta$ is low) or $\gamma$ is low) then (Q is safety).

Rule 3: if ( $\alpha$ is medium) or ( $\beta$ is medium) or ( $\gamma$ is medium) then ( $Q$ is danger).

Rule 4: if ( $\alpha$ is medium) or ( $\beta$ is high) or ( $\gamma$ is high) then ( $Q$ is warning).

Rule 5: if ( $\alpha$ is not high) or ( $\beta$ is medium) or ( $\gamma$ is not high) then ( $Q$ is warning).

Rule 6: if ( $\alpha$ is not high) or ( $\beta$ is not high) or ( $\gamma$ is medium) then ( $Q$ is warning).

\subsection{An example of if-then fuzzy inference rules based on Matlab simulation}

A simulation result using Fuzzy inference system(FIS) of Matlab fuzzy logic toolbox of and selecting Mamdani type fuzzy inference system to establish the crane real-time safety evaluation model is shown in Fig.6.
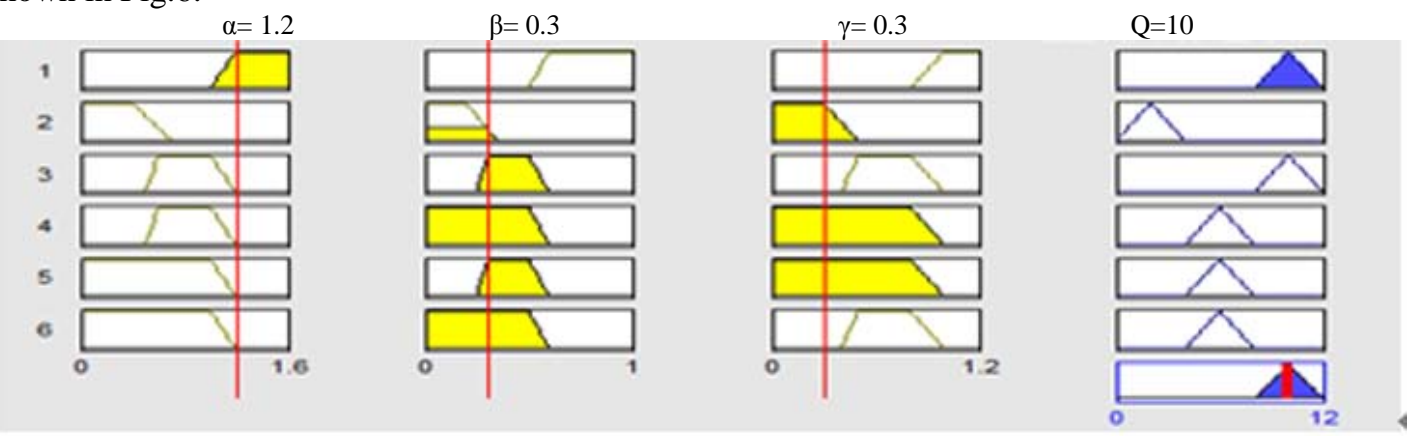

Figure6. Example of if-then inference rules in Matlab simulation

As shown in Fig6,if $\alpha=1.2$ (high), $\beta=0.3$ (low), $\gamma=0.3$ (low), according to rule 1 , crane real time safety grade $\mathrm{Q}$ is danger, and simulation results $\mathrm{Q}=10$. After defuzzification is done,safety state of the crane is danger, so the theoretical analysis and simulation results are consistent.

\subsection{Matlab simulation analysis of Mamdani type fuzzy inference system}

Matlab is used to simulate abovesecurity evaluation model of Mamdani type fuzzy inference. Simulation result shows that the system can reflect real timesafety status of the crane according to real time evaluation index data. The influence of three input variables $\alpha, \beta$ and $\gamma$ on safety grade $Q$ is shown in Fig.7.The reasoning result of crane safety state depends on the value of each input variable,Fig.7 shows that smaller the fluctuation range of $\alpha, \beta$, and $\gamma$ is, smaller the $\mathrm{Q}$ is. The curved 
surface also shows that it is difficult to use accurate mathematical model description, and fuzzy reasoning provides an effective solution for this kind of application.

Q

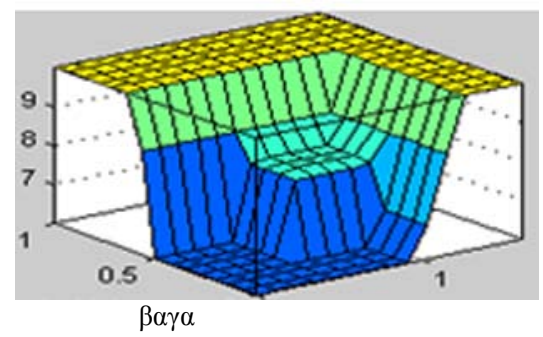

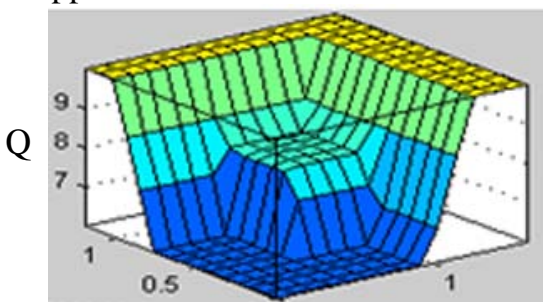

Figure 7. (a)Effect of $\alpha$, $\beta$ on $Q(b)$ Effect of $\alpha, \gamma$ on $Q$

\section{Field test and conclusion}

A real 400 ton crawler crane as experimental object is used to verify the fuzzy model. Corresponding sensors and other hardware facilities are installed to designated location of the crane. The HMI system mainly composed of real-time data display module and real-time safety grade evaluation module, and developed based on above Mamdani type fuzzy inference evaluation model.

The operator operates the crane with controlling speeds of crane movement, rotation, lifting and so on according to the evaluation results. In this way, the safety risk caused by operator himself is reduced. The test results show that the established evaluation index system and security model are feasible, a warning can be indicated immediately, and the evaluation based on result of language description can reflect real-time safety status of crane lifting process directly.

\section{Acknowledgement}

This work was financially supported by Guangdong Provincial Science and Technology Plan Project (2014A010103027), and Huizhou Municipal Science and Technology Plan Project (2015B020005007).

\section{References}

1. Hongzhi Chen, Jian Chen, and Peng Li, Research of the crane boom bending monitoring technology based on image matching algorithm[J], Hoisting and Conveying Machinery, 2015(3):99-102.

2. ifmelectronic Co. Ltd., CR0505 System Manual, http://www.ifm.com.

3. Guoquan Liu and Jian Chen, Design of monitoring system of hook of crane based on ZigBee wireless sensor network, Hoisting and Conveying Machinery, 2015(4):41-45.

4. Mamdani E H, Assilian S, An experiment in linguistic synthesis with a fuzzy logic controller[J]. International Journal of ManMachine Studies, 1975, 7(1): 1-13.

5. JijianXie, ChengpingLiu, Fuzzy mathematics method and its application[M], Wuhan: Huazhong University of Science and Technology Press, 2006. 